\title{
Serum ghrelin levels in patients with Behcet's disease
}

\author{
Ilker Erden¹, Haydar Uçak², Betül Demir ${ }^{3}$, Demet Cicek ${ }^{3}$, Selma Bakar Dertlioğlu³, Suleyman Aydin', Savas Ozturk ${ }^{5}$ \\ ${ }^{1}$ Department of Dermatology, Elazig Education and Research Hospital, Elazig, Turkey \\ 2Department of Dermatology, Faculty of Medicine, Dicle University, Diyarbakir, Turkey \\ ${ }^{3}$ Department of Dermatology, Faculty of Medicine, Firat University, Elazig, Turkey \\ ${ }^{4}$ Department of Medical Biochemistry and Clinical Biochemistry (Firat Hormones Research Group), Faculty of Medicine, Firat University, \\ Elazig, Turkey \\ ${ }^{5}$ Department of Dermatology, Faculty of Medicine, Balıkesir University, Balıkesir, Turkey
}

Adv Dermatol Allergol 2016; XXXIII (6): 450-456

DOI:10.5114/ada.2016.63884

\begin{abstract}
Introduction: Behcet's disease (BD) is a chronic, relapsing, systemic vasculitis of unknown etiology. Aim: To measure serum ghrelin levels in BD patients and healthy controls and to investigate its association with metabolic syndrome (MetS).

Material and methods: Thirty BD patients and 30 healthy individuals were enrolled in the study. Ghrelin levels were measured in blood samples using ELISA.

Results: The mean serum ghrelin level in BD patients $(28.57 \pm 14.04)$ was significantly lower compared to healthy controls $(40.72 \pm 23.21)(p=0.01)$. The mean serum ghrelin level in BD patients who had MetS $(24.18 \pm 12.73)$ was lower compared to BD patients who did not have MetS $(30.77 \pm 14.45)$, but this difference was not significant $(p>$ 0.05).

Conclusions: Ghrelin levels were lower in BD patients compared to healthy controls. There was no association between reduced ghrelin levels and MetS; however, there was a negative correlation between ghrelin levels and disease activity.
\end{abstract}

Key words: Behcet's disease, ghrelin, metabolic syndrome, insulin resistance.

\section{Introduction}

Behcet's disease (BD) is a multisystemic, chronic, inflammatory disease characterized with recurrent oral and genital ulcerations and uveitis attacks [1, 2]. While the disease etiopathogenesis is not fully known, emphasis is given to genetic factors, microbial agents, and endothelial cell dysfunction [1, 3, 4]. Recent studies suggest that the prevalence of metabolic syndrome in patients with BD is higher compared to the normal population [5].

Ghrelin was discovered by Japanese scientists in 1999 as an endogenous ligand for growth-hormone secretagogue (GHS-R), which is capable of inducing growth hormone secretion from the pituitary gland [6]. Ghrelin is a 28-amino acid, lipopeptide hormone secreted primarily from the stomach fundus. In addition to the stomach, ghrelin is also synthesized in the hypothalamus, pituitary gland, thyroid gland, small intestine, kidneys, heart, $\alpha$-, $\beta$-, and $\varepsilon$-cells of the pancreas, the central nervous system, lung, placenta, gonads, immune system, breasts, and teeth [6-10]. Ghrelin's effect on appetite has been experimentally documented [7]. Immunohistochemical analyses have shown that ghrelin is endogenously expressed in $\alpha$-, $\beta$-, and $\varepsilon$-cells of pancreatic islets, while GSH-R1a is primarily expressed in pancreatic $\alpha$ - and $\beta$ cells [11]. These findings indicate that the regulation of ghrelin and insulin levels are highly related. In addition, plasma ghrelin levels are low in obesity, and have been regarded as an important finding of metabolic syndrome, just like hyperinsulinemia and insulin resistance [12, 13].

\section{Aim}

This study aims to investigate ghrelin levels and the association between ghrelin and MetS in patients with $\mathrm{BD}$ in light of all available information.

\section{Material and methods}

Patient selection and patient characteristics

This study was carried out on 30 BD patients who were followed up at the Dermatology Department of the

Address for correspondence: Haydar Uçak, Department of Dermatology, Faculty of Medicine, Dicle University, TR21070 Diyarbakir, Turkey, phone: +90 412 2488001/4334, fax: +90 412 2488440, e-mail: ucak23@mynet.com Received: 23.10.2015, accepted: 12.11.2015. 
Faculty of Medicine at Firat University and at the Dermatology Department of Elazig Teaching and Research Hospital, and 30 healthy individuals were included as the control group. One-to-one interviews were conducted with 30 patients (15 males and 15 females) diagnosed based on International Behcet's Disease Study Group Criteria [14], and the patients' ages, genders, disease duration, medications, and history of smoking were recorded. BD-associated symptoms and/or findings (oral ulcer, genital ulcer, eye involvement, arthralgia, arthritis, acneiform skin lesions, erythema nodosum-like lesions, thrombophlebitis, gastrointestinal involvement, neurological involvement, and pathergy test results) were recorded. Thirty healthy individuals (15 males and $15 \mathrm{fe-}$ males), who were admitted to the hospital for annual check-ups with no systemic, dermatologic, rheumatologic, or neurological diseases (including family history) and did not use alcohol, recreational substances, or any medication, were included in the control group.

Exclusion criteria: 1) age < 18 years, 2) pregnancy, 3) acute or chronic infection, 4) acute or chronic neurological disorders (excluding Neuro-Behcet's disease), 5) polycystic ovary syndrome or amenorrhea, 6) hyperthyroidism or hypothyroidism.

\section{Study plan}

All enrolled patients were analyzed for age, gender, age of onset, duration of disease, history of smoking and alcohol use, height, weight, body mass index (BMI), and waist circumference. The BD-associated symptoms and/ or findings (oral ulcer, genital ulcer, eye involvement, arthralgia, arthritis, acneiform skin lesions, erythema nodosum-like lesions, thrombophlebitis, gastrointestinal involvement, neurological involvement, and pathergy test results) were recorded. Patients' symptoms and/or findings were recorded as follows: active, inactive (previous), or not observed. Ghrelin levels may be affected by many metabolic factors, and therefore, patients and controls had similar BMI to decrease the different metabolic factors in BD patients.

Body mass index was calculated as weight/height $\left(\mathrm{kg} / \mathrm{m}^{2}\right)$ and metabolic syndrome was diagnosed in the presence of central obesity in addition to two or more criteria of the International Diabetes Foundation [15]. In addition, insulin resistance was calculated based on the homeostasis model assessment of insulin resistance (HOMA-IR) formula. Cases with a HOMA-IR index of $>3.2$ were diagnosed to have insulin resistance [16].

\section{Collection and storage of biological samples}

As ghrelin is a peptide hormone and can be broken down by proteases, aprotinin (500 Kallikrein units per $\mathrm{ml}$ ) was added to plain biochemistry tubes before the collection of blood samples from the participants to prevent proteolysis [17]. Blood samples were collected between 9:00 am and 10:00 am after an overnight fast to avoid any effects associated with circadian rhythm. Serum ghrelin levels were analyzed using a human ghrelin kit (Cat. No. A05106; SPI-Bio, Montigny-le-Bretonneux, France) by the enzyme-linked immunosorbent assay (ELISA) method according to the manufacturer's instructions. According to the kit's supplier, the intra- and interassay coefficients of variation (CV) for this kit are $<7 \%$ and $<8.1 \%$, respectively.

\section{Statistical analysis}

SPSS version 12.0 (SPSS, Chicago, IL) was used for statistical analyses. Data were presented as mean \pm SD. Independent samples $t$-test and Mann-Whitney $U$-test were used to compare groups. In all analyses, $p$-values $<0.05$ were considered statistically significant.

\section{Results}

Thirty BD patients and 30 healthy controls who were admitted to the Department of Dermatology of the Faculty of Medicine at Firat University and the Dermatology Department of Elazig Teaching and Research Hospital were included in the study. The age range in $\mathrm{BD}$ patients (25-54) and the control group (21-64) was similar. The disease duration ranged between 1 and 17 years in BD patients, while the mean disease period was $5.96 \pm 4.4$ years. Female/male (F/M) ratio was $1: 1$ in both groups. There was no significant difference in age, gender, and $\mathrm{BMI}$ distribution between the groups $(p>0.05)$. The demographic and clinical features of patient and control groups are presented in Table 1.

Family history was positive in three patients with $\mathrm{BD}$ (10\%), while none of the healthy controls had a history of $\mathrm{BD}$ in their families. The symptoms and/or findings of patients with $\mathrm{BD}$ were recorded as follows: active, inactive (previous), or not observed. The BD patients with at least two active systemic findings/symptoms were considered active patients. Based on this classification, 14 (46.7\%) patients were active, and 16 (53.3\%) were inactive. The

Table 1. Clinical findings of patient and control groups

\begin{tabular}{lccc}
\hline Parameter & $\begin{array}{c}\text { Behcet's } \\
\text { disease }\end{array}$ & Control & $P$-value \\
\hline$N$ & 30 & 30 & \\
\hline Gender (M/F) & $15 / 15$ & $15 / 15$ & $>0.05$ \\
\hline Age $^{*}$ year] & $36.66 \pm 7.9$ & $37.20 \pm 11.3$ & $>0.05$ \\
\hline BMI $^{\star}\left[\mathrm{kg} / \mathrm{m}^{2}\right]$ & $24.08 \pm 1.57$ & $24.47 \pm 1.63$ & $>0.05$ \\
\hline BMI score* $^{*}$ & $2.23 \pm 0.43$ & $2.33 \pm 0.47$ & $>0.05$ \\
\hline $\begin{array}{l}\text { Waist circumference } \\
{[\mathrm{cm}]}\end{array}$ & $85.53 \pm 8.37$ & $87.33 \pm 9.58$ & $>0.05$ \\
\hline
\end{tabular}

${ }^{\star}$ Mean \pm standard deviation. 
Table 2. Clinical properties of Behcet's disease patients

\begin{tabular}{|c|c|c|c|c|c|c|c|c|c|c|c|c|c|}
\hline No. & Age & Gender & OU & GU & Eye & Joint & ENLL & Acne & DVT & CS & GIS & $\mathrm{NI}$ & Pathergy \\
\hline 1 & 37 & $M$ & 2 & 2 & 0 & 0 & 0 & 0 & 0 & 0 & 0 & 2 & 2 \\
\hline 2 & 30 & $\mathrm{~F}$ & 1 & 1 & 0 & 2 & 1 & 0 & 0 & 0 & 0 & 0 & 0 \\
\hline 3 & 40 & $\mathrm{~F}$ & 2 & 2 & 1 & 0 & 0 & 2 & 0 & 0 & 0 & 0 & 0 \\
\hline 4 & 39 & M & 1 & 1 & 1 & 0 & 0 & 0 & 0 & 0 & 0 & 0 & 1 \\
\hline 5 & 39 & M & 1 & 1 & 2 & 2 & 0 & 0 & 0 & 0 & 0 & 0 & 0 \\
\hline 6 & 32 & $\mathrm{~F}$ & 1 & 1 & 1 & 0 & 0 & 0 & 0 & 0 & 0 & 0 & 1 \\
\hline 7 & 45 & M & 1 & 1 & 0 & 0 & 2 & 0 & 0 & 0 & 0 & 0 & 1 \\
\hline 8 & 45 & $\mathrm{~F}$ & 2 & 1 & 1 & 0 & 0 & 0 & 0 & 0 & 0 & 0 & 0 \\
\hline 9 & 34 & M & 2 & 0 & 2 & 2 & 0 & 1 & 0 & 0 & 0 & 0 & 2 \\
\hline 10 & 29 & $\mathrm{~F}$ & 2 & 1 & 0 & 2 & 1 & 0 & 0 & 1 & 0 & 0 & 0 \\
\hline 11 & 38 & $\mathrm{~F}$ & 1 & 1 & 0 & 0 & 2 & 0 & 2 & 0 & 0 & 0 & 0 \\
\hline 12 & 28 & $M$ & 1 & 1 & 1 & 0 & 1 & 0 & 0 & 0 & 0 & 0 & 0 \\
\hline 13 & 54 & $\mathrm{~F}$ & 2 & 2 & 2 & 2 & 0 & 2 & 0 & 0 & 0 & 1 & 2 \\
\hline 14 & 39 & $\mathrm{~F}$ & 2 & 2 & 2 & 2 & 0 & 0 & 0 & 0 & 0 & 0 & 0 \\
\hline 15 & 36 & $M$ & 1 & 1 & 1 & 1 & 0 & 0 & 1 & 0 & 0 & 0 & 0 \\
\hline 16 & 52 & $M$ & 1 & 1 & 1 & 0 & 0 & 0 & 0 & 0 & 0 & 0 & 0 \\
\hline 17 & 31 & $\mathrm{~F}$ & 2 & 2 & 1 & 0 & 0 & 0 & 1 & 0 & 0 & 0 & 0 \\
\hline 18 & 43 & $M$ & 1 & 1 & 1 & 1 & 0 & 0 & 0 & 0 & 0 & 0 & 0 \\
\hline 19 & 28 & $M$ & 2 & 1 & 0 & 0 & 1 & 1 & 0 & 0 & 0 & 0 & 0 \\
\hline 20 & 54 & $\mathrm{~F}$ & 2 & 2 & 1 & 0 & 0 & 0 & 0 & 0 & 0 & 0 & 2 \\
\hline 21 & 31 & $M$ & 1 & 1 & 0 & 1 & 0 & 0 & 0 & 0 & 0 & 0 & 1 \\
\hline 22 & 27 & $M$ & 2 & 1 & 1 & 2 & 1 & 0 & 0 & 2 & 0 & 0 & 0 \\
\hline 23 & 31 & $\mathrm{~F}$ & 1 & 1 & 0 & 0 & 0 & 0 & 0 & 0 & 0 & 0 & 1 \\
\hline 24 & 25 & $M$ & 2 & 2 & 1 & 0 & 0 & 0 & 0 & 0 & 0 & 0 & 2 \\
\hline 25 & 34 & $\mathrm{~F}$ & 1 & 1 & 0 & 0 & 0 & 0 & 0 & 0 & 0 & 0 & 1 \\
\hline 26 & 31 & $\mathrm{~F}$ & 1 & 2 & 1 & 0 & 0 & 0 & 2 & 0 & 0 & 0 & 0 \\
\hline 27 & 42 & $\mathrm{~F}$ & 1 & 1 & 1 & 1 & 0 & 1 & 0 & 0 & 0 & 0 & 0 \\
\hline 28 & 40 & $M$ & 2 & 1 & 1 & 0 & 1 & 0 & 0 & 0 & 0 & 0 & 0 \\
\hline 29 & 39 & $\mathrm{~F}$ & 1 & 2 & 1 & 2 & 1 & 0 & 0 & 1 & 0 & 0 & 2 \\
\hline 30 & 54 & $M$ & 1 & 1 & 1 & 1 & 0 & 0 & 0 & 0 & 1 & 0 & 0 \\
\hline
\end{tabular}

CS - cardiovascular system, DVT - deeep vena thrombosis, ENLL - eritema nodosum like lesion, F-female, GIS-gastrointestinal system, GU - genital ulcer, $\mathrm{M}$ - male, $\mathrm{NI}$ - neurologic involvement, $\mathrm{OU}$-oral ulcer, $\mathrm{O}$ - never happened, 1 -inactive, 2 - active.

clinical features of patients with $\mathrm{BD}$ are presented in Table 2. Nine (30\%) BD patients used colchicine, and 9 (30\%) BD patients used systemic corticosteroids, and immunosuppressive agents including azathioprine and cyclosporine in addition to colchicine. Twelve (40\%) patients were not on any treatment.

The mean serum ghrelin level in BD patients (28.57 $\pm 14.04)$ were significantly lower compared to healthy controls $(40.72 \pm 23.21)(p=0.01)$. Similarly, the mean serum glucose level in BD patients $(80.10 \pm 1.84)$ was significantly lower compared to healthy controls (88.06 \pm 10.45 , $p=0.008$ ) (Table 3 and Figure 1).
An evaluation of the patients and the controls for metabolic syndrome (MetS) was observed in 12 (40\%) BD patients and 12 (40\%) controls. The mean serum ghrelin level in BD patients with MetS $(24.18 \pm 12.73)$ was lower compared to BD patients without MetS $(30.77 \pm 14.45)$, but this difference was not significant $(p>0.05)$. A grouping of BD patients for $\mathrm{IR}$ and/or MetS presence found no significant differences in serum ghrelin levels between the groups. However, an evaluation of all patients and controls demonstrated that the mean serum ghrelin level in BD patients with IR and/or MetS (28.64 \pm 17.90 ) was significantly lower compared to BD patients without IR and/or MetS (38.93 \pm 20.52$)(p=0.03)$ (Figure 2). 
An examination of the association between disease activity and serum ghrelin, glucose, insulin, and C-peptide levels showed that the mean serum ghrelin level in active BD patients $(26.97 \pm 15.52)$ was lower compared to inactive BD patients $(29.98 \pm 12.95)$, but the difference between the groups was insignificant. Similarly, there was no significant difference in serum C-peptide and insulin levels between active and inactive BD patients $(p<0.05)$. However, the mean serum glucose level in active BD patients $(72.14 \pm 7.57)$ was significantly lower compared to inactive BD patients $(85.12 \pm 12.51, p=0.001)$ (Figure 3$)$.

Table 3. Laboratory findings of patient and control groups

\begin{tabular}{|c|c|c|c|}
\hline Parameter & $\begin{array}{l}\text { Behcet's } \\
\text { disease }\end{array}$ & Control & $P$-value \\
\hline $\begin{array}{l}\text { Glucose* } \\
{[\mathrm{mg} / \mathrm{dl}]}\end{array}$ & $79.06 \pm 12.25$ & $86.86 \pm 10.84$ & 0.01 \\
\hline $\begin{array}{l}\text { Triglycerides* } \\
\text { [mg/dl] }\end{array}$ & $133.76 \pm 53.38$ & $131.70 \pm 83.31$ & $>0.05$ \\
\hline $\begin{array}{l}\text { LDL- } \\
\text { cholesterol* }^{*} \\
{[\mathrm{mg} / \mathrm{dl}]}\end{array}$ & $114.43 \pm 26.94$ & $108.67 \pm 25.49$ & $>0.05$ \\
\hline $\begin{array}{l}\text { HDL- } \\
\text { cholesterol* }^{*} \\
{[\mathrm{mg} / \mathrm{dl}]}\end{array}$ & $45.44 \pm 11.54$ & $46.28 \pm 12.17$ & $>0.05$ \\
\hline $\begin{array}{l}\text { Total } \\
\text { cholesterol* } \\
{[\mathrm{mg} / \mathrm{dl}]}\end{array}$ & $170.76 \pm 39.81$ & $181.00 \pm 21.66$ & $>0.05$ \\
\hline $\begin{array}{l}\text { Insülin* } \\
{[\mu \mid U / m l]}\end{array}$ & $8.39 \pm 6.83$ & $9.01 \pm 3.85$ & $>0.05$ \\
\hline $\begin{array}{l}\text { C-peptid* } \\
{[\mathrm{ng} / \mathrm{ml}]}\end{array}$ & $2.57 \pm 1.27$ & $2.11 \pm 0.74$ & $>0.05$ \\
\hline $\begin{array}{l}\text { HOMA-IR } \\
\text { values* }\end{array}$ & $1.66 \pm 1.39$ & $1.99 \pm 0.93$ & $>0.05$ \\
\hline $\begin{array}{l}\text { Ghrelin* } \\
{[\mathrm{pg} / \mathrm{ml}]}\end{array}$ & $28.57 \pm 14.04$ & $40.72 \pm 23.21$ & 0.01 \\
\hline
\end{tabular}

*Mean \pm standard deviation.

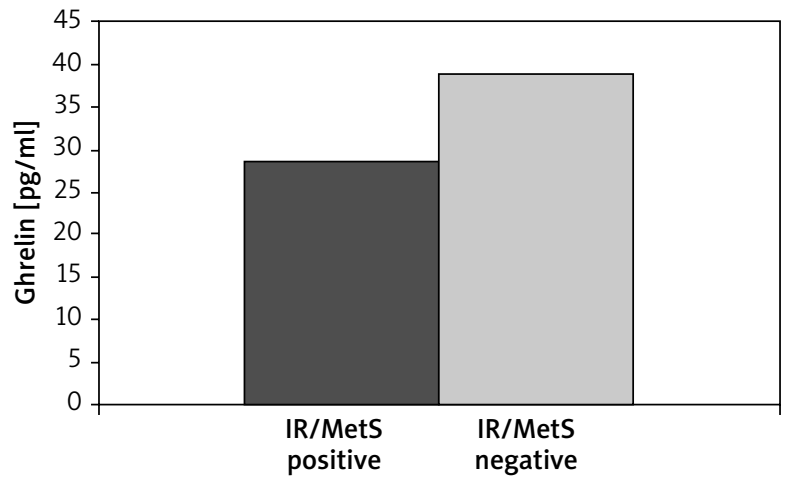

Figure 2. Mean serum ghrelin levels in groups with IR/MetS positive and IR/MetS negative
Behcet's disease may progress with multisystemic involvements, and different system involvements can be observed in each patient. Given that the oral mucosa is an important site regarding involvement in $\mathrm{BD}$, the study evaluated the serum ghrelin levels in BD patients with and without active oral involvement. The mean serum ghrelin level was $30.50 \pm 16.73$ in BD patients with oral involvement and $27.10 \pm 11.91$ in BD patients without oral involvement; however, there was no significant difference between the groups $(p>0.05)$.

There was a positive correlation between ghrelin level and BMI, and ghrelin level and glucose level in BD patients $(r=0.48, p<0.05 ; r=0.55, p<0.001$, respectively). On the other hand, there was a negative correlation between ghrelin level and disease activity $(r=-0.45$, $p<0.05)$.

\section{Discussion}

Various metabolic functions of ghrelin have been defined since its discovery as a molecule that induces growth hormone secretion from the pituitary gland.

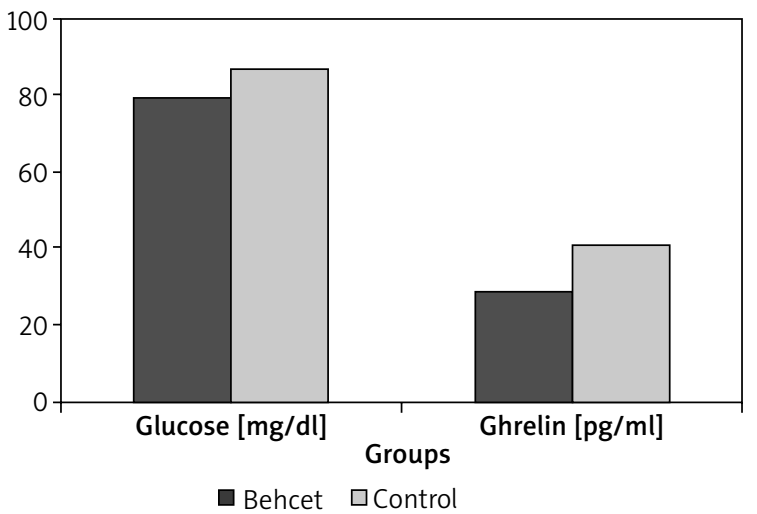

Figure 1. Mean serum ghrelin and glucose levels in groups

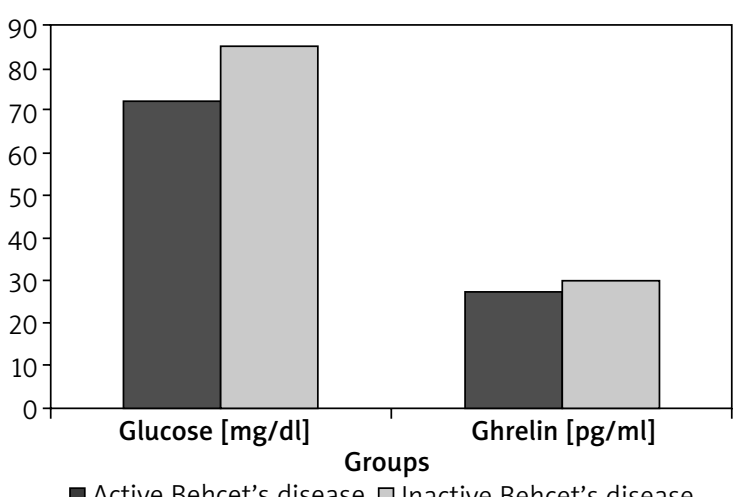

Figure 3. Mean serum ghrelin and glucose levels in active and inactive groups 
Throughout the day, ghrelin levels increase in case of fasting, and decrease during fullness. Circulating ghrelin levels have been shown to have a regulatory effect on body weight in the long term [18]. A negative correlation between ghrelin levels and body weight has been shown in patients who are on low-calorie diets, have cancer-associated anorexia, and anorexia nervosa. It has also been reported that increased weight in patients with anorexia nervosa reduces ghrelin levels [19].

Serum ghrelin levels are low in patients with type 2 diabetes or insulin resistance [20]. Moreover, studies have shown that saliva ghrelin levels in type 2 diabetes patients are low, and this could serve as an important parameter to monitor the course of diabetes [10]. In fact, some authors suggest that a low ghrelin level is an indicator of MetS [21]. According to a study on premenopausal and non-diabetic women, there was a strong association between fasting ghrelin levels and subcutaneous lipid mass, while insulin resistance showed a negative correlation with ghrelin levels [20]. On the other hand, ghrelin levels were normal in children with type I diabetes [22]. Studies have shown that ghrelin levels are lower in obese individuals compared to those who are underweight. Weight loss through diet caused an increase in ghrelin levels [23].

In the present study, serum ghrelin levels were significantly lower in BD patients compared to healthy individuals. In addition, there was a positive correlation between serum ghrelin level and BMI in BD patients. This finding was consistent with various studies that have shown that serum ghrelin levels decrease in obese patients. Interestingly, while serum glucose levels were within the normal range, BD patients had significantly lower serum glucose levels compared to healthy controls. In addition, there was a positive correlation between serum ghrelin levels and serum glucose levels. These findings were interesting, as ghrelin levels are expected to increase during fasting, and decrease during fullness; however, both serum glucose and ghrelin levels were lower in the present study, which could be explained as follows: different studies have shown that ghrelin, glucose and insulin might display different behaviors in metabolic events, especially in case of external stimuli. Acute ghrelin administration induces hyperglycemia, and reduces insulin levels, both in humans and rodents [24]. In this case, ghrelin and glucose levels are elevated at the same time, while later on, the decrease in circulating ghrelin levels implies that glucose levels will also decrease in parallel. In addition, studies have shown that insulin decreases ghrelin levels. Following the gradual induction of hyperinsulinemia (1.2 and $4 \mu \mathrm{U} / \mathrm{kg} / \mathrm{s}$ ), ghrelin levels during normoglycemia decreased by $17 \%, 27 \%$, and $33 \%$, respectively. The finding that the induction of hyperglycemia during normoglycemia decreases ghrelin levels indicates that insulin might play an important role in regulating ghrelin levels [25]. On the other hand, when hyperglycemia was induced with $50 \mathrm{~g}$ of glucose administration, plasma ghrelin levels were reduced to the minimum level 30 min after the plasma glucose level returned to the normal level [26].

There is only a single study in the literature on serum ghrelin levels in BD patients. Koca et al. [27] carried out a study on 29 BD patients, and did not determine any significant difference in serum ghrelin levels between BD patients and healthy controls.

Polycystic ovary syndrome (PCOS) is accompanied by insulin resistance, and studies on PCOS patients have contradictory findings. Waśko et al. [28] found that PCOS patients had high ghrelin levels compared to the control group, reporting a negative correlation between ghrelin level and BMI, and insulin level. Another study reported that ghrelin levels in PCOS patients were not significantly different compared to the control group [29], while Pagotto et al. [30] reported that PCOS patients had lower ghrelin levels compared to obese patients. Schöfl et al. [31] found that ghrelin levels were lower in PCOS patients compared to healthy, obese, or underweight individuals. This study revealed that BD patients with MetS had lower ghrelin levels compared to controls, although this difference was not significant. Due to the insufficient number of patients with insulin resistance, no comparison could be made between BD patients with and without insulin resistance. However, when patients and controls are considered together, the study found that serum ghrelin levels were significantly lower in individuals who had IR and/or MetS compared to individuals who did not have IR and/or MetS.

Studies have shown variable serum ghrelin levels in chronic inflammatory diseases [32-34]. An increase in ghrelin levels has been shown in the case of active chronic inflammatory diseases such as ankylosing spondylitis, ANCA-dependent vasculitis, celiac disease, and inflammatory bowel disease [32, 34]. According to another study, serum ghrelin levels were higher in patients with psoriasis compared to healthy controls; however, this difference was not significant [35]. On the other hand, Otero et al. [33] reported that ghrelin levels were decreased in rheumatoid arthritis (RA) patients. Koca et al. found that serum ghrelin levels were lower in RA patients compared to $\mathrm{BD}$ patients. The authors argued that the decrease in ghrelin levels could be due to the high-dose corticosteroid treatment [26].

Neutrophil-mediated cytokine levels are elevated in BD patients [1, 3]. Ghrelin is well known to suppress the secretion of proinflammatory cytokines and chemokines, and the growth of inflammatory cells [36]. On the other hand, studies have shown that cytokines directly suppress ghrelin synthesis [37]. In this study, significantly lower ghrelin levels in BD patients, and also in active BD patients could be explained by elevated cytokine levels in BD patients. Few studies reported a negative correlation between ghrelin levels and the severity of psoriasis $[35,38]$. Similarly, Koca et al. [27] stated that inflamma- 
tion and cytokines could be a reason for lower ghrelin levels in RA. The same study also emphasized the fact that lower ghrelin levels may worsen the prognosis of RA [27]. This study found lower ghrelin levels in patients with active BD; however, this reduction was not statistically significant. On the other hand, the study detected a negative correlation between the ghrelin level and disease activity, and the ghrelin level and glucose level.

Studies on mice have shown that plasma ghrelin levels were higher in females compared to males. Similarly, studies on humans also showed that plasma ghrelin levels were higher in females [39]. In this study, ghrelin levels were also higher in women, and this finding was consistent with other studies in the literature.

\section{Conclusions}

Ghrelin is a recently-discovered anabolic hormone with side effects including ghrelin growth, appetite, fat accumulation, and increased gluconeogenesis and functions such as energy consumption and storage in the brain and in peripheral tissues. This study found a significant decrease in ghrelin levels in BD patients compared to healthy controls. The decrease in ghrelin levels could not be correlated with MetS; however, the study found a negative correlation between ghrelin levels and disease activity. Therefore, the researchers consider that ghrelin levels are reduced in BD due to a mechanism different than the development of MetS and inflammatory processes might be effective in this regard. Larger studies focusing on the relation between the immune system, severity of BD and ghrelin levels may be useful to clarify the etiopathogenesis of the disease and to improve activation markers for the patients with $\mathrm{BD}$.

\section{Conflict of interest}

The authors declare no conflict of interest.

\section{References}

1. Gül A. Behcet's disease: an update on the pathogenesis. Clin Exp Rheum 2001; 19: 6-12.

2. Woźniacka A, Jurowski P, Omulecki A, et al. Behçet’s disease leaves the silk road. Postep Derm Alergol 2014; 31: 417-20.

3. Direskeneli H. Behcet's disease: infectious aetiology, new autoantigens and HLA-B51. Ann Rheum Dis 2001; 60: 9961002.

4. Rizzi R, Bruno S, Dammacco R. Behcet's disease: an immunemediated vasculitis involving vessels of all sizes. Int J Clin Lab Res 1997; 27: 225-32.

5. Yalçın B, Gür G, Artüz F, et al. Prevalence of metabolic syndrome in Behcet disease: a case-control study in Turkey. Am J Clin Dermatol 2013; 14: 421-5.

6. Kojima M, Hosoda H, Date Y, et al. Grelin is a growth hormone-releasing acylated peptide from stomach. Nature 1999; 402: 656-9.
7. Korbonits M, Goldstone AP, Gueorguiev M, et al. Grelin a hormone with multiple functions. Front Neuroend 2004; 25: 27-68.

8. Kojima M, Kangawa K. Grelin: structure and function. Physiol Rev 2005; 85: 495-522.

9. Aydin S, Aydin S, Ozkan Y, et al. Grelin is present in human colostrum, transitional and mature milk. Peptides 2006; 27: 878-82.

10. Aydin S, Ozercan IH, Geckil H, et al. Grelin is present in human teeth. J Biochem Mol Biol. 2007; 40: 368-72.

11. Dezaki K, Sone H, Yada T. Ghrelin is a physiological regulator of insulin release in pancreatic islets and glucose homeostasis. Pharmacol Ther 2008; 118: 239-49.

12. McLaughlin T, Abbasi F, Lamendola C, et al. Plasma ghrelin concentrations are decreased in insulin-resistant obese adults relative to equally obese insulin-sensitive controls. J Clin Endocrinol Metab 2004; 89: 1630-5.

13. Cong WN, Golden E, Pantaleo N, et al. Ghrelin receptor signaling: a promising therapeutic target for metabolic syndrome and cognitive dysfunction. CNS Neurol Disord Drug Targets 2010; 9: 557-63.

14. International Study Group for Behcet's Disease. Criteria for diagnosis of Behcet's disease. Lancet 1990; 335: 1078-80.

15. Sidiropoulos PI, Karvounaris SA, Boumpas DT. Metabolic syndrome in rheumatic diseases: epidemiology, pathophysiology, and clinical implications. Arthritis Res Ther 2008; 10: 207.

16. Kondo N, Nomura M, Nakaya Y, et al. Association of inflammatory marker and highly sensitive $\mathrm{C}$ reactive protein with aerobic exercise capacity, maximum oxygen uptake and insulin resistance in healthy middle aged volunteers. Circ J 2005; 69: 452-57.

17. Stengel A, Keire D, Goebel M, et al. The RAPID method for blood processing yields new insight in plasma concentrations and molecular forms of circulating gut peptides. Endocrinology 2009; 150: 5113-8.

18. Chan JL, Bullen J, Lee JH, et al. Ghrelin levels are not regulated by recombinant leptin administration and/or three days of fasting in healthy subjects. J Clin Endocrinol Metab 2004; 89: 335-43.

19. Tolle V, Kadem M, Bluet-Pajot MT, et al. Balance in ghrelin and leptin plasma levels in anorexia nervosa patients and constitutionally thin women. J Clin Endocrinol Metab 2003; 88: 109-16.

20. Pöykkö SM, Kellokoski E, Hörkkö S, et al. Low plasma grelin is associated with insulin resistance, hypertension, and the prevalence of type 2 diabetes. Diabetes 2003; 52: 2546-53.

21. Ukkola O, Poykko SM, Antero Kesaniemi Y. Low plasma grelin concentration is an indicator of the metabolic syndrome. Ann Med 2006; 38: 274-9.

22. Bideci A, Camurdan MO, Cinaz P, et al. Grelin, IGF-I and IGFBP-3 levels in children with type 1 diabetes mellitus. J Pediatr Endocrinol Metab 2005; 18: 1433-9.

23. Cummings DE. Grelin and the short- and long-term regulation of appetite and body weight. Physiol Behav 2006; 89: 71-84.

24. Broglio F, Arvat E, Benso A, et al. Ghrelin, a natural GH secretagogue produced by the stomach, induces hyperglycemia and reduces insulin secretion in humans. J Clin Endocrinol Metab 2001; 86: 5083-6.

25. Anderwald-Stadler M, Krebs M, Promintzer M, et al. Plasma obestatin is lower at fasting and not suppressed by insulin in insulin-resistant humans. Am J Physiol Endocrinol Metab 2007; 293: 1393-8. 
26. Nakagawa E, Nagaya N, Okumura H, et al. Hyperglycaemia suppresses the secretion of ghrelin, a novel growth-hormone-releasing peptide: responses to the intravenous and oral administration of glucose. Clin Sci 2002; 103: 325-8.

27. Koca SS, Ozgen M, Aydin S, et al. Ghrelin and obestatin levels in rheumatoid arthritis. Inflammation 2008; 31: 329-35.

28. Waśko R, Komarowska H, Warenik-Szymankiewicz A, et al. Elevated ghrelin plasma levels in patients with polycystic ovary syndrome. Horm Metab Res 2004; 36: 170-3.

29. Orio F Jr, Lucidi P, Palomba S, et al. Circulating ghrelin concentrations in the polycystic ovary syndrome. J Clin Endocrinol Metab 2003; 88: 942-5.

30. Pagotto U, Gambineri A, Vicennati V, et al. Plasma ghrelin, obesity, and the polycystic ovary syndrome: correlation with insulin resistance and androgen levels. J Clin Endocrinol Metab 2002; 87: 5625-9.

31. Schöfl C, Horn R, Schill T, et al. Circulating ghrelin levels in patients with polycystic ovary syndrome. J Clin Endocrinol Metab 2002; 87: 4607-10.

32. Peracchi $M$, Conte D, Terrani $C$, et al. Circulating ghrelin levels in celiac patients. Am J Gastroenterol 2003; 98: 2474-8.

33. Otero M, Nogueiras R, Lago F, et al. Chronic inflammation modulates ghrelin levels in humans and rats. Rheumatology 2004; 43: 306-10.

34. Toussirot E, Streit G, Nguyen NU, et al. Adipose tissue, serum adipokines, and ghrelin in patients with ankylosing spondylitis. Metabolism 2007; 56: 1383-9.

35. Ozdemir M, Yüksel M, Gökbel H, et al. Serum leptin, adiponectin, resistin and ghrelin levels in psoriatic patients treated with cyclosporin. J Dermatol 2012; 39: 443-8.

36. Dixit VD, Schaffer EM, Pyle RS, et al. Ghrelin inhibits leptinand activation-induced proinflammatory cytokine expression by human monocytes and T cells. J Clin Invest 2004; 114: $57-66$

37. Dixit VD, Taub DD. Ghrelin and immunity: a young player in an old field. Exp Gerontol 2005; 40: 900-10.

38. Ucak H, Demir B, Cicek D, et al. Metabolic changes and serum ghrelin level in patients with psoriasis. Dermatol Res Pract 2014; 2014: 175693.

39. Gualillo O, Caminos JE, Kojima M, et al. Gender and gonadal influences on ghrelin mRNA levels in rat stomach. Eur J Endocrinol 2001; 144: 687-90. 